\title{
CBTC Simulation Platform Design and Study
}

\author{
Jikang Xu' ${ }^{1}$, Lijun Chen', Weimin Gao ${ }^{2}$, Minjie Zhao ${ }^{2}$ \\ ${ }^{1}$ College of Urban Rail Transit, Shanghai University of Engineering Science, Shanghai, China \\ ${ }^{2}$ Shanghai Rail Transit Maintenance Support Limited Company, Shanghai, China \\ Email: jikangxu@sues.edu.cn
}

Received 8 August 2015; accepted 14 September 2015; published 17 September 2015

Copyright (C) 2015 by authors and Scientific Research Publishing Inc.

This work is licensed under the Creative Commons Attribution International License (CC BY). http://creativecommons.org/licenses/by/4.0/

cC) (i) Open Access

\begin{abstract}
In order to train and test the functionality of the CBTC signal system, this paper designs simulation platform's architecture and function module based on the CBTC principle firstly. Then, it detailedly discusses the main content and key algorithms about train module and trackside module. Finally, it builds CBTC simulation test platform, used for training of rail transit signal related specialized student and detecting the main function of actual CBTC system based on Shanghai Metro Line 9.
\end{abstract}

Keywords

CBTC, Simulation Platform, Test

\section{Introduction}

With the development of computer technology and communication technology, communication-based train control system (CBTC) has become the mainstream train control technology in the field of domestic and international rail transportation industry [1]. However, the current rail traffic signal related major in college lacks of teaching and training equipment according to the related to CBTC system, poor association of the teaching curriculum and practical signal system. At the same time, due to the lack of a unified CBTC test platform, universities, research institutes and other the third party safety testing institutions cannot detect the safety and reliability of CBTC system provided by the signal provider. Therefore, it's imminent to design and research of CBTC simulation and test platform for meeting the requirement of teaching training and signal system safety testing.

\section{Simulation Platform Design}

\subsection{Principle}

According to the IEEE 1474.1, CBTC is continuous train automatic control system [2] by vehicle and ground 
safety processor, based on the high precision train positioning, high-capacity train-wayside communication technology. Actual CBTC system architecture [3] is shown in Figure 1.

CBTC system is mainly composed of the following subsystems: ATS subsystem, including ATS server and multiple ATS station; trackside subsystem, including zone controller subsystem and interlocking subsystem; vehicle subsystem and the communication between each subsystems [4]. It ensures the train operation effectively and safely through the collaboration between these subsystems.

\subsection{Module Design}

According to the principle of CBTC, the simulation platform is divided into the following modules: trackside module, train module and ATS module, as shown in Figure 2. The trackside module and the train module are integrated in the ATS server through simulation technology [5]. The two modules can run either on the same hardware or independent of ATS server.

\subsubsection{Trackside Module}

Trackside module simulates the functions of zone controller and interlock, mainly including the route processing and authorization calculation [6]. When the ATS client requests route, validation of the route will be processed by trackside module. If the route is established (switch position, block status etc.) to meet the requirements, route establish successful. Then, it calculates and updates the train moving authorization by other functions. The specific route processing model of state machine is shown in Figure 3.

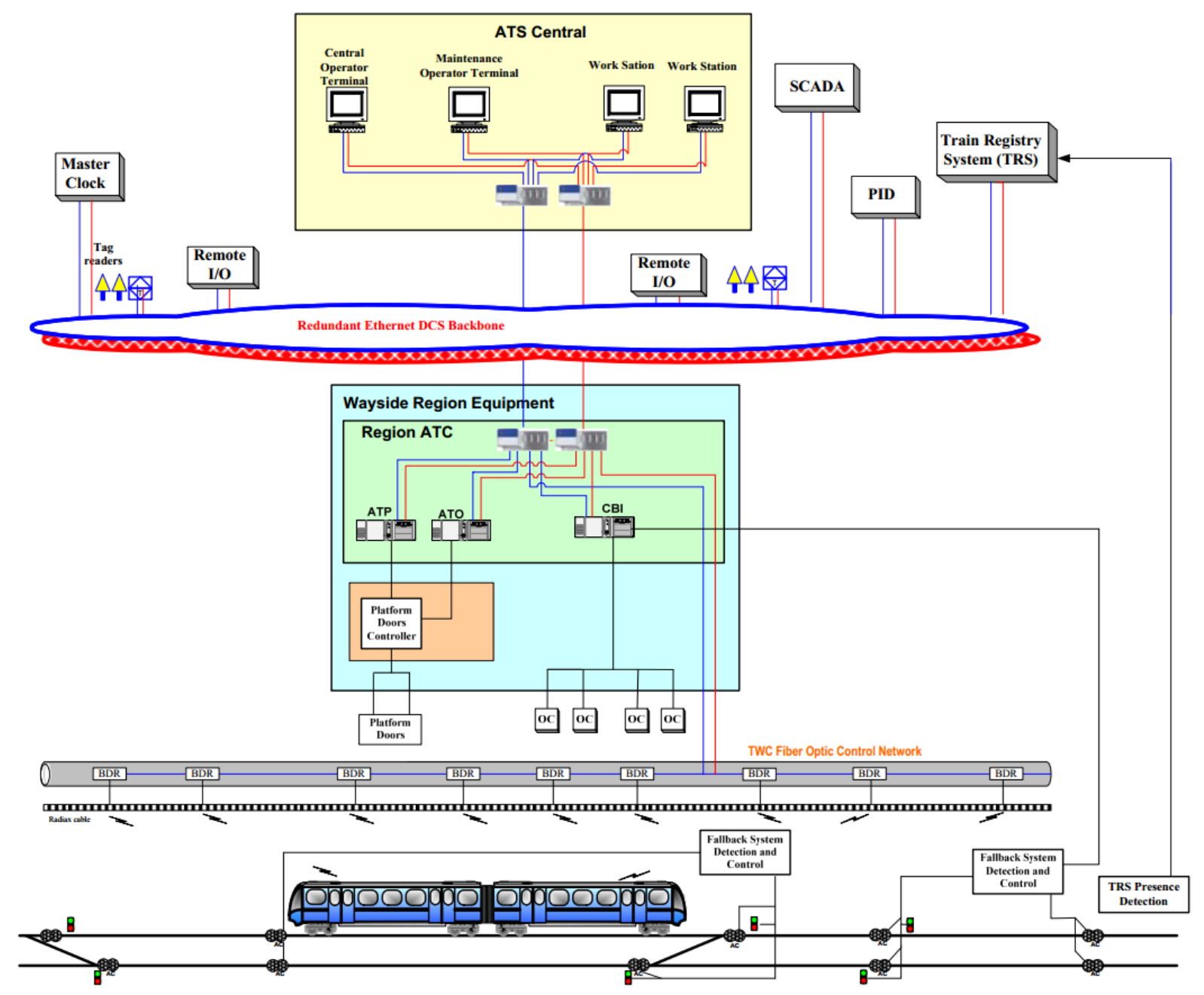

Figure 1. CBTC system architecture. 


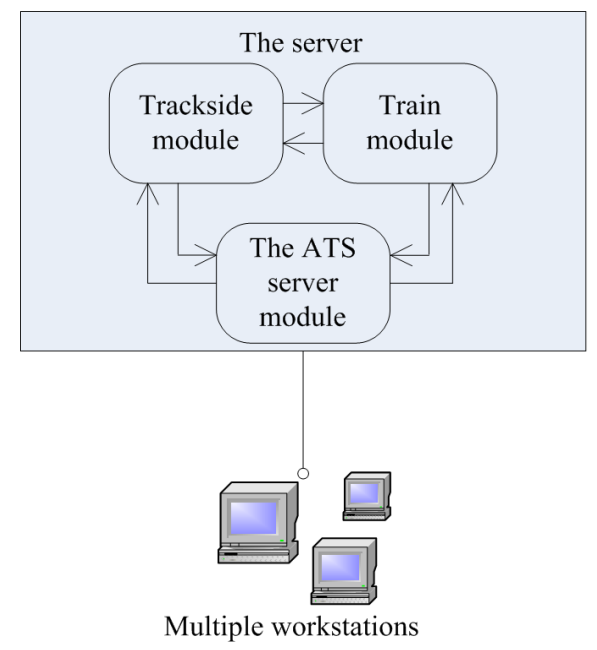

Figure 2. CBTC simulation platform architecture.

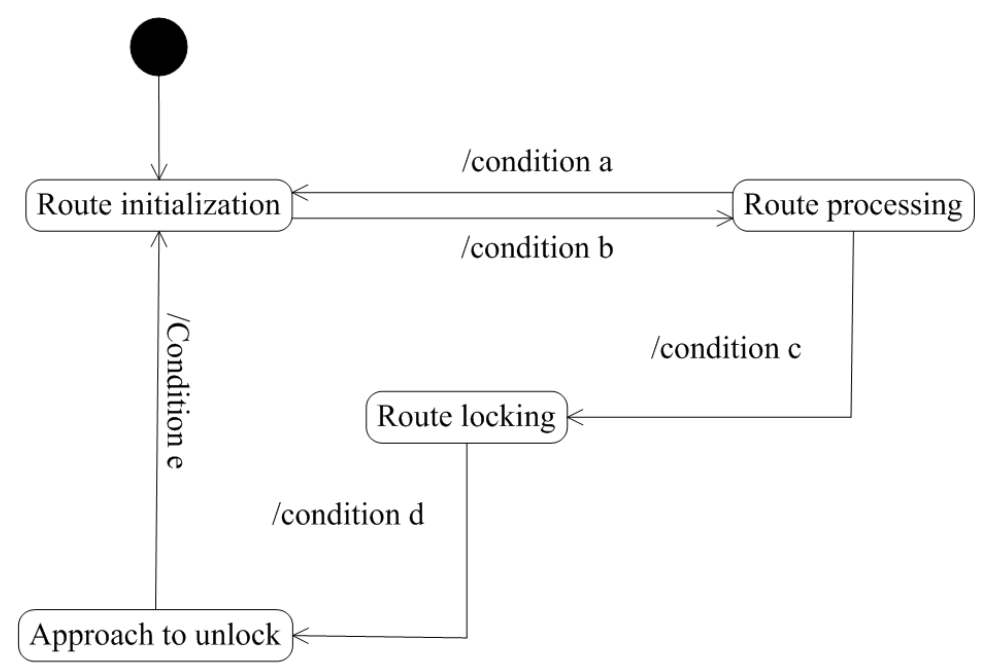

Condition a: route is not reasonable.

Condition b: ATS route request is reasonable, to meet the conditions.

Condition $\mathrm{c}$ : the conditions of establishing reasonable route, route for success.

Condition d: complete the use of Train route, Soon leaves the route area..

Condition e: approach to unlock success, return to the initial state.

Figure 3. Route state machine model.

Authorized processing model of state machine, as shown in Figure 4.

\subsubsection{Train Module}

CBTC has higher train positioning accuracy. Therefore, train module mainly simulates the train movement process, including the accurate position of the train, speed calculation. In calculating the train speed and position, this module adopts following model, considering the influence of grade to the train acceleration.

$$
\begin{aligned}
& a_{i}=g * \sin (\theta) \\
& v_{i}=v_{i-1}+a_{i} * \Delta t \\
& s_{i}=s_{i-1}+\left(v_{i-1} * \Delta t+\frac{a * \Delta t^{2}}{2}\right)
\end{aligned}
$$


$a_{i}$ - the moment $i$ of train acceleration;

$g$-acceleration of gravity, taking $9.8 \mathrm{~m} / \mathrm{s}^{2}$;

$\theta$-the slope of line angle;

$v_{i}$-the cycle $i$ of train speed;

$\Delta t$-cycle time;

$s_{i}$ - the cycle $i$ of train position.

At the same time, in order to update the train front and rear position, this train module uses the following algorithm. As shown in Figure 5.

Step 1: Train moves forward/backward on the track, and update strain front and rear position respectively. It calculates the train front position, going to Step 2, the rear position, going to Step 3.

Step 2: If the train front position crossed the track boundary, going to Step 4; otherwise, update train front position, going to Step 3 .

Step 3: If the train rear position crossed the track boundary, going to Step 5; otherwise, update the train rear position, going to Step 5 .

Step 4: Update the train front edge information and the train front offset at edge.

Step 5: Update the train rear edge information and the train rear offset at edge.

Step 6: Train location update success, the end.

Figure 6 is the flow chart about train position update.

\section{Result}

It uses tools of QT and C++ to develop a simulation platform based on CBTC as, CBTC system's ATS client interface as Shanghai Metro Line 9 as example, shown in Figure 7. The platform mainly provides with CBTC system's training and test function.

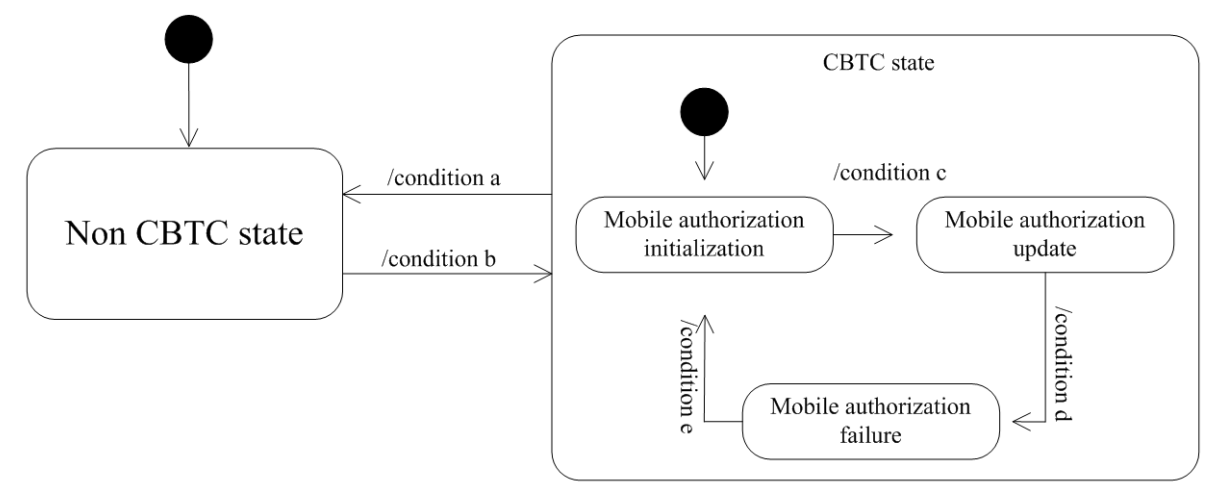

Condition a: Train left CBTC area.

Condition b: Train enter CBTC area.

Condition c: received route information and position information of train.

Condition d: train-ground communication fault.

Condition e: train-ground communication recovery.

Figure 4. Authorized computing model of state machine.

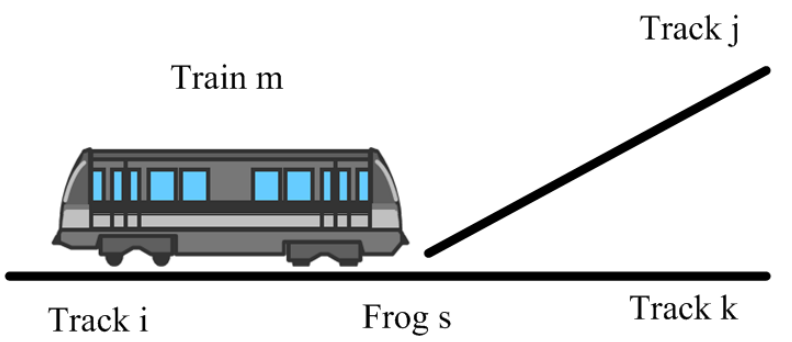

Figure 5. Train position update diagram. 


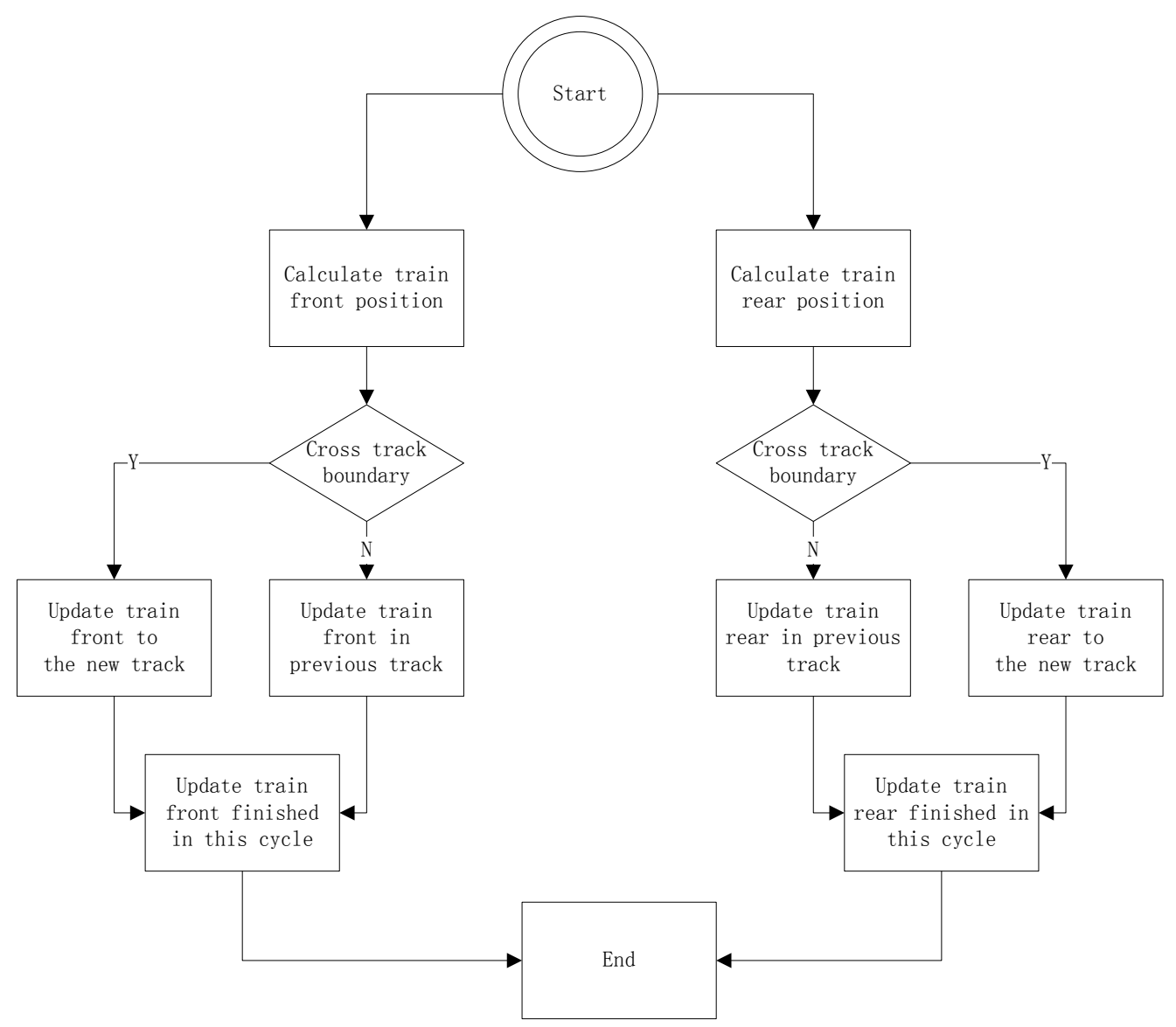

Figure 6. Train position update flow chart.

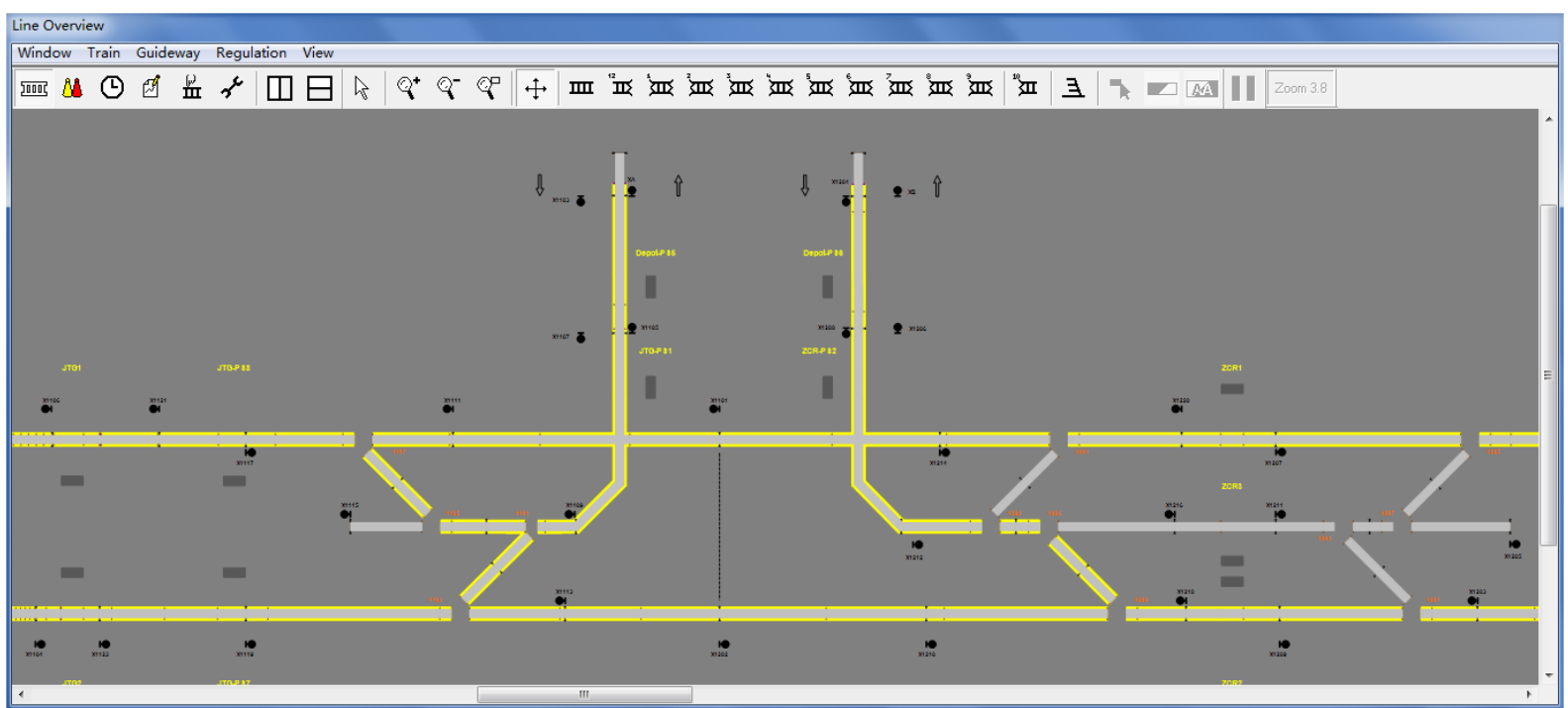

Figure 7. CBTC simulation platform based on Shanghai Metro Line 9.

\subsection{Training Function}

This platform includes the following experiments, shown in Table 1 . The students can fully understand the CBTC system's functions, including solving process in the common failure case of CBTC system. 
Table 1. CBTC simulation platform experiments.

\begin{tabular}{ccc}
\hline Training programs & Properties (verification/synthesis/design/innovation) & Hours \\
\hline Route control experiment & Synthesis & 8 \\
Train control experiment & Synthesis & 8 \\
Switch control experiment & Synthesis & 8 \\
Schedule function test experiment & Synthesis & 8 \\
Platform control experiment & Synthesis & 8 \\
LMA experiment & Synthesis & 8 \\
Fault simulation experiment & Synthesis & 12 \\
\hline
\end{tabular}

These experiments are not only suitable for students of rail transit control major, but also suitable for students of rail transit operation and management.

\subsection{Detection Function}

The test platform uses common interface, and it can test the actual signal system's subsystem function, including the vehicle subsystem and trackside subsystem test, as follows:

1) Detection external signal function from vehicle controller

It simulates the electrical interface between vehicle controller unit and vehicle system, and the external interface of the vehicle controller. The reliability, integrity, and security about signal input and output of the vehicle controller can be detected through this function.

2) Safety function detection of vehicle controller unit

Safety function detection of vehicle controller mainly include: the safety train speed and safety position; the train over speed protection function; the safety interval function of the train; the train slide down protection function etc.

3) Automatic driving function detection

Detection the reliability about automatic train operation. For example, the precision and accuracy of alignment the station alignment while the train operation under various conditions.

\section{Conclusion}

The CBTC platform can not only simulate the main functions of CBTC system under normal circumstances, but also simulate emergency process functions. Through the construction of the platform and the related experimental training, it helps students learn the architecture and function of CBTC system and improve the teaching effect. At the same time, with the development of the platform, it also can be applied in the safety assessment and detection of metro signal system.

\section{Acknowledgements}

The authors gratefully acknowledge the support of the following projects:

1) The Training Project of Youth College Teachers of Shanghai Municipal Education Commission (ZZGJD13042).

2) The research of general technology about interface between vehicle and ATC signal (JS-BZ13R006).

\section{References}

[1] Yin, X.Z., Li, L. and Wang, W. (2013) The Research and Implementation of CBTC Simulation and Training System. Railway Signal and Communication, 49, 141-144.

[2] Chen, X.X., Wang, D. and Huang, H. (2011) The Simulation Test Platform’s Design of CBTC System. Railway Computer Application, 8, 50-56.

[3] (2015) Bombardier Type CITYFLO 650 Automatic Train Operation CONTROL System [EB/OL]. Wikipedia. http://zh.wikipedia.org/

[4] Mu, Z.H. (2012) CBTC Simulation Teaching System Research and Development of Urban Rail Transit. Chinese Rail- 
way, 7, 77-79.

[5] Chen, W.H. (2010) The CBTC Simulation System Applicable to ATS Testing and Training. Railway Signal and Communication, 3, 28-30.

[6] IEEE Vehicular Technology Society (2005) IEEE Standard for Communication-Based Train Control (CBTC). Performance and Functional Requirements, 5-6. 